\title{
The impact of Hurricane Maria on out-migration from Puerto Rico: Evidence from Facebook data
}

\author{
Monica Alexander*1, Kivan Polimis ${ }^{2}$, and Emilio Zagheni ${ }^{3}$ \\ ${ }^{1}$ University of Toronto \\ ${ }^{2}$ MAANA \\ ${ }^{3}$ Max Planck Institute for Demographic Research
}

\begin{abstract}
Natural disasters such as hurricanes can cause substantial population outmigration. However, the magnitude of population movements is difficult to estimate using only traditional sources of migration data. We utilize data obtained from Facebook's advertising platform to estimate out-migration from Puerto Rico in the months after Hurricane Maria. We find evidence to indicate a $17.0 \%$ increase in the number of Puerto Rican migrants present in the US over the period October 2017 to January 2018. States with the biggest increases were Florida, New York and Pennsylvania, and there were disproportionately larger increases in the 15-30 age groups and for men compared to women. Additionally, we find evidence of subsequent return migration to Puerto Rico over the period January 2018 to March 2018. These results illustrate the power of complementing social media and traditional data to monitor demographic indicators over time, particularly after a shock, such as a natural disaster, to understand large changes in population characteristics.
\end{abstract}

\section{Introduction}

Understanding migration patterns, including emigration destinations and the characteristics of those who moved and those who are left behind, has important implications for designing policy to best help those in need. It is particularly relevant to be able to track changes in population after disasters of large-scale environmental events. A

\footnotetext{
*monica.alexander@utoronto.ca
} 
recent notable example is Hurricane Maria, which hit Puerto Rico in September 2017. While the initial official death toll was reported by the government as 64, this was recently revised up to 2,975 (Santos-Burgoa et al., 2018), after independent researchers observed that a higher death count was more likely than the initial total (Kishore et al., 2018; Santos-Lozada and Howard, 2018). Additionally, lack of basic amenities, like power and clean water, still remain a problem in many parts of this US territory almost one year after the hurricane (Kishore et al., 2018).

Besides the impact on mortality, natural disasters can also cause substantial outmigration from the location affected. For example, approximately 1.5 million New Orleans residents evacuated around the time of Hurricane Katrina in 2005, and two years later more than a third of the pre-hurricane residents of New Orleans had not returned to the city (Fussell et al., 2010). Initial estimates for Puerto Rico suggest that out-migration was roughly $6 \%$ of the total population (Echenique and Melgar, 2018). A decrease in the size of the total population of this magnitude has important implications for both the origin and the receiving locations. In addition to that, if not properly accounted for, large flows of migrants skew estimates of mortality, as they affect the denominator for mortality rates, i.e. population counts across different groups.

Although data on migration are key for demographic estimation and projections — and to understand societal implications of events like natural disasters - they often do not exist, and those that do exist are typically not produced in a timely manner. For example, in the United States, there is no publicly available dataset on monthly migration movements between states and outlying territories. The American Community Survey (ACS) provides details on birthplace and place of residence one year before the interview. This is a good resource to estimate migration rates by state and other demographic characteristics. However, ACS estimates are only produced annually, with no information available about changes in migration stocks from month 
to month. In addition, ACS data are published with a delay: the 2017 estimates had been released only in late October 2018. As such, other sources of data are often used to track short-term migration changes. For example, previous work has used mobile phone data, as well as change of address and school enrollment changes, or flight passenger data, to track movements from Puerto Rico to the US after Hurricane Maria (Echenique and Melgar, 2018; Meléndez and Hinojosa, 2017; Rayer, 2018). However, even when some approximate estimates are produced indirectly using these types of data, basic demographic characteristics of migrants are typically not available.

Recently, population scientists have started to study social media data that include rich demographic information. For example, many social networking sites offer a glimpse into the demographic characteristics of their users via their advertising platforms. As a matter of fact, Facebook can be thought of as a large 'digital census' that is regularly updated. However, a major issue with these data is that they are not representative of underlying populations, and thus can lead to biased inference. Notwithstanding that, previous research has shown that biases can be modeled and filtered out using statistical approaches, in particular when social media data can be 'calibrated' with data from representative surveys (Yildiz et al., 2017; Zagheni et al., 2014, 2017).

In this paper, we offer both a methodological contribution to the literature that uses data from social media advertisement platforms for demographic research, and a substantive contribution to the literature on the impact of Hurricane Maria on Puerto Rico. From the methodological point of view, previous research using Facebook data for advertisers focused on assessing biases by comparing, in a statistical sense, social media data and representative samples in order to provide timely estimates of changes in annual migration stocks (Zagheni et al., 2017). For this paper, we do not have 'ground truth' information to calibrate social media data, in part because the American Community Survey is not designed to provide estimates of movements at a time 
granularity finer than 1-year intervals. In order to address this issue, we propose an approach that relies on the difference-in-differences estimation procedure to adjust for known biases in the Facebook population compared to the broader population.

From the substantive point of view, we add to the literature on the effects of Hurricane Maria on Puerto Rico by offering estimates of migration that, unlike those that come from flight passenger data or other indirect sources, are broken down by age and sex, and include an assessment of return migration. In particular, we estimated that there was a $17.0 \%$ increase in the number of Puerto Rican migrants present in continental US over the period from October 2017 to January 2018, corresponding to an increase of around 185,200 people. States with the biggest increases were Florida, New York and Pennsylvania, and there were disproportionately larger increases in the 15-30 age groups, and for men compared to women. Data from subsequent periods suggest that nationally, there was a $1.8 \%$ decrease of Puerto Ricans present in continental US from January to March 2018, suggesting evidence for return migration to Puerto Rico.

For our purposes, Facebook data are particularly well-suited to studying Puerto Rican migration due to the large proportion of the population that uses Facebook on a regular basis in Puerto Rico (Rosado, 2017). Importantly, although Facebook data have a number of limitations, in the context of estimating population movement from Puerto Rico after Hurricane Maria, Facebook is a key data source that we believe should be taken seriously.

The remainder of the paper is structured as follows. In the next section, we describe how data were collected from the Facebook's Advertising Platform and the characteristics of these data, including potential data issues. Then we describe the methods used for estimating migration of Puerto Ricans to continental US. Section 4 offers key results of migration estimates by state, age and sex. Finally, we discuss the implications and limitations of this analysis. 


\section{Data}

Facebook has developed a targeted advertising platform, called Ads Manager, which uses a graphical user interface to allow advertisers to target specific audiences. The dimensions that can be targeted include information directly reported by Facebook users, such as age or sex, and information indirectly inferred from the use of the Facebook platform or affiliated websites, such as location and behavioral interests. Importantly in the context of this research, we can choose to target audiences based on where they have lived before. Before launching a marketing campaign, an advertiser can select a variety of characteristics (e.g., Puerto Ricans living in California, who are female, and aged 30-35) and get an estimate of the 'potential reach' (monthly active users) for each subgroup. These estimates can be obtained, in a programmatic way, for a variety of different groups, broken down, for instance, by US state of residence, age, and sex.

It is the estimates of users who lived in Puerto Rico and moved to continental US, by age and sex that we use to track migration stocks over time. These estimates can be obtained before the launch of an advertisement, and as such are obtained free of charge. We use the Ads Manager application, Facebook's Marketing API, to extract estimates of potential reach over time programmatically via the Python module pySocialWatcher (Araujo et al., 2017).

As part of a broader project on using social media in demographic research, we started data collection in January 2017. This data collection is twofold for each wave, state-level estimates of all Facebook users (by age, sex, and gender) as well as statelevel estimates of 28 immigrant groups by age and sex. ${ }^{1}$ Since beginning the data collection, we have collected a new wave of data every 2-3 months (wave 1: January 2017; wave 2: April 2017; wave 3: June 2017; wave 4: October 2017; wave 5: January 2018; wave 6: March 2018). 
For the purposes of this project, wave 4 overlaps with the period when Hurricane Maria impacted Puerto Rico. As such, we use the change in the Facebook potential reach from wave 4 to wave 5 as the basis of our estimates of out-migration. We also use the change from wave 5 to wave 6 to assess evidence for subsequent migration of Puerto Ricans in the next three months. Note that the Facebook data provide information on the change in Puerto Rican migrants on continental US, rather than a change in the native population in Puerto Rico. As such, we are assuming that increases in Puerto Rican migrants on continental US correspond to out-migration from Puerto Rico, and decreases in migrants in continental US correspond to in-migration (return migration) to Puerto Rico.

\subsection{Data Challenges}

An important challenge with Facebook data is that the population of Facebook users is not representative of the broader population. In particular, the age distribution of Facebook users is on average younger than the broader population. We compare the age distribution of Puerto Rican migrants in the first wave of the Facebook data with the age distribution of Puerto Rican migrants in the 2017 5-year ACS. Figure 1 shows the two age distributions for the nine states with the largest populations of Puerto Rican migrants. In all states, the ACS has an older age distribution, while the Facebook distributions are concentrated in the age groups less than 30 years. While some states, such as Texas and Florida, have similarly shaped age distributions, the shapes of the age distributions are very different in most states.

[Figure 1 about here.]

Similarly, the sex distribution of Puerto Rican migrants across the US differs between the Facebook and ACS data (Table 1). For example, while the male-to-female 
ratio of Puerto Ricans in California is around 1.3 in the ACS, the Facebook data suggest fewer men and more women, with a sex ratio of 0.94 . In contrast, the sex ratio in Connecticut is around 0.85 in the ACS, while the Facebook data suggest many more men, with a sex ratio of 1.35. Similar patterns to Connecticut are seen in most of the top states, with Facebook suggesting higher proportions of men than ACS. Given these observations, along with the observation of different age distributions, appropriate adjustments are needed to extract meaningful information from non-representative Facebook samples.

[Table 1 about here.]

A second major source of bias is related to incomplete information about the algorithms that Facebook uses internally in order to estimate migrants (Facebook referred to migrants as 'expats' or 'ex-pats' in the Marketing API at the time of data collection for this study). Estimates of migrant users in the Facebook data appear to vary over time (wave), independently of underlying changes in migrant stocks, because of rounding and possibly because of adjustments of the algorithms internally used. For example, there was a marked decrease of approximately $10 \%$ in the size of all migrant populations from wave 3 to wave 4 , followed by an approximate $10 \%$ increase going from wave 4 to wave 5 (see Supplementary materials). These changes are independent of migrant origin and do not appear to be linked to any broad-scale migration trends. It seems more likely that these large changes are related to Facebook's method of estimating 'population at reach'. If we only looked at changes in the Puerto Rican population over waves, without accounting for potential platform-wide changes, we would overestimate the increase in migrants after Hurricane Maria. 


\section{Methods}

In order to address the data challenges described above, our method of estimating the extent of out migration from Puerto Rico involved two main steps: using Differencein-Differences to get estimates of the percent change in migrants, and then applying these to ACS data to get population estimates by demographic subgroups.

\subsection{Estimating percentage change in migration using Difference- in-Differences}

As mentioned in the previous section, there are two main issues with using the Facebook data in its unadjusted form to estimate migration: firstly, the population of Facebook is not representative, and secondly, there are fluctuations in the data over time that appear to be independent of actual migration events. To overcome these issues, we use Difference-in-Differences (DiD) to estimate the percent change in Puerto Rican migrants in continental US after Hurricane Maria. The method of DiD

involves comparing the difference in the size of a group of interest (in our case, Puerto Rican migrants in the US) before and after an event (in our case, Hurricane Maria) to the difference in the size of a 'control' group before and after the same event. By considering the change in the group of interest relative to a control group, any changes that have occurred that are common to both groups are differenced out. In addition, we consider the percent change before and after, rather than the raw population increase or decrease, to reduce issues related to Facebook being non-representative.

In particular, define $\pi_{g}$ to be the proportional change in Puerto Rican migrants over the period October 2017 to January 2018 (i.e. waves 4 and 5 in our data) for group of interest $g$. The group $g$ might be all Puerto Rican migrants at the national level, or stratified by any age, sex and state group, for example Puerto Rican migrants 
aged $30-34$ in Florida. We estimate $\pi_{g}$ as

$$
\hat{\pi}_{g}=\frac{P_{g}^{P R}(5)-P_{g}^{P R}(4)}{P_{g}^{P R}(4)}-\frac{P_{g}^{C}(5)-P_{g}^{C}(4)}{P_{g}^{C}(4)}
$$

where $P_{g}^{P R}(w)$ refers to the population of Puerto Rican migrants in group $g$ at wave $w$ (where $w=4$ or 5 ), and $P_{g}^{C}(w)$ refers to the population in the control group for the same group and wave.

We compare the change in Puerto Rican migrants over time to the change in the control group, that is migrants from all other origins collected in the Facebook data (see endnote 1), with the exception of Mexico. This comparison assumes that migration decisions by those in the control group would not have been affected by the hurricane. We remove Mexico from the control group to avoid large fluctuations in the Mexican migrant population due to reasons other than Hurricane Maria. We used DiD to estimate changes in the Puerto Rican migrant population for the whole US, by state, age and sex. Standard errors were calculated based on the Binomial approximation to the Normal distribution.

Note that there are important assumptions made when using this methodological approach. Firstly, DiD inherently assumes that, had Hurricane Maria not occurred, the relationship between the two migrant groups would have remained the same. One way to assess the validity of this assumption is to check trends in the migrant groups before the hurricane. As shown in the Supplementary materials, the trends are generally parallel before October, thus supporting the validity of our assumption. Secondly, by tracking changes in the Facebook data, we are assuming that the population of Puerto Ricans in Facebook had a similar migration behavior in response to Hurricane Maria to Puerto Ricans in the general population. For example, if those with a Facebook account were more likely to migrate than those without, then this approach would overestimate the extent of out-migration. It is difficult to check the validity of this 
assumption; however, given the large-scale use of Facebook in Puerto Rico (Rosado, 2017), differential use of the social media website by characteristics like income and education - which are likely to also be correlated with propensity to migrate - would not be very prevalent.

It should be noted that to avoid issues with rounding in the Facebook data, for the state-by-state analysis, we only consider states that had a total Puerto Rican migrant population in the Facebook data of at least 18,000 people. This corresponds to at least 1,000 people in each of the 18 age by sex groups (2 sexes and 9 five-year age groups from 15-60).

\subsection{Converting percentages to numbers using ACS}

The DiD approach allows us to obtain estimates of the percent increase or decrease of the migrant groups we are interested in. As a second step, we take the estimated percentage changes from $\mathrm{DiD}$ and multiply these by the relevant populations as reported in the ACS, to get an estimate for the change in the number of migrants. For example, the change in the number of Puerto Rican migrants pre- and post- Hurricane Maria is estimated as

$$
\hat{M}_{g}=\hat{\pi}_{g} \cdot P_{g}^{A C S}
$$

where $\hat{M}_{g}$ is the estimated change in the number of Puerto Ricans in group $g, \hat{\pi}_{g}$ is

the estimated proportional change in Puerto Rican migrants in that group, and $P_{g}^{A C S}$ is the number of Puerto Rican migrants in group $g$ as reported in the ACS. A similar approach is taken for all the relevant age, state and sex groups of interest. 


\section{Results}

Over the period from October 2017 to January 2018, at the national level we estimate a $17.0 \%$ increase in the number of Puerto Rican migrants present in the US. This is equivalent to an increase of approximately 185,200 people, corresponding to approximately $5.6 \%$ of the total population of Puerto Rico (US Census Bureau, 2018).

On a state-by-state basis, the states with the largest existing populations of Puerto Rican migrants also had the largest increases after Hurricane Maria (Table 2, Figure 2). Florida had the largest percentage increase in Puerto Rican migration, increasing $21.6 \%$ over the period October 2017 to January 2018. This corresponded to an increase of around 65,400 people. Pennsylvania, New York, Connecticut and Massachusetts also had substantial increases of 8,000-15,000 migrants.

[Table 2 about here.]

[Figure 2 about here.]

\subsection{Changes by age and sex}

Figure 3 shows the proportional change in each five-year age group aged 15-60 in the nine states with the largest Puerto Rican migrant populations. In general, there is a noticeable increase in the 15-30 age range. This suggests those people moving away from Puerto Rico after the hurricane were those in the working age groups.

[Figure 3 about here.]

We define the sex ratio of migrants as the number of men divided by the number of women. All of the nine states have sex ratios greater than one, meaning that there were more men than women. In the period after the hurricane, the sex ratios remained fairly stable, suggesting that the number of women migrating compared to men did not 
change much. However, as shown in Table 3, there was an increase in the sex ratios for states such as Florida and Texas, suggesting that the distribution of migrants shifted to include more men. The opposite was true for Pennsylvania, which saw a $5.3 \%$ decrease in the sex ratio, suggesting an increase in women.

[Table 3 about here.]

\subsection{Evidence of return migration since January 2018}

In addition to assessing changes in Puerto Rican population in the period from October 2017 to January 2018, we also looked at the change in migrant stocks from January 2018 to March 2018 to assess the extent to which migration out of Puerto Rico that occurred in the three months following the hurricane was counteracted with return migration in subsequent months. Note that here we are assuming that an observed decrease in the Puerto Rican population in continental US would likely correspond to Puerto Ricans returning home after the hurricane.

The results suggest that at the national level there was a $1.8 \%$ decrease in the Puerto Rican population in continental US, corresponding to around 19,500 people. In terms of specific states, there was a $7.1 \%$ decrease in the Puerto Rican population in Florida, corresponding to a decrease in population of around 21,500 people (Table 4). Other states such as Massachusetts and Connecticut saw decreases of 3 to $4 \%$, corresponding to decreases of around 2,000-4,000 people. In contrast, other states such as California and New Jersey show evidence of continuing increases in the Puerto Rican population.

[Table 4 about here.] 


\section{Discussion}

Data obtained from Facebook's advertising platform highlight the substantial outmigration from Puerto Rico in the three months following Hurricane Maria. The data suggest a $17 \%$ increase in Puerto Rican migrant population in continental US in the three months following the Hurricane, corresponding to around 185,200 people. People who moved were disproportionately in the younger working age groups and men. Additionally, we saw evidence of return migration to Puerto Rico, as suggested by a $1.8 \%$ decrease in Puerto Ricans in the continental US from January to March 2018 (Table 4).

The estimated outflows for the total population and population by state are in line with previous studies that utilized other sources of data, including cell phone records (Echenique and Melgar, 2018), flight passenger data (Rayer, 2018), port authority data (Santos, 2018), and forecasts from the American Community Survey (Meléndez and Hinojosa, 2017). For example, at the national level using ACS data, Meléndez and Hinojosa (2017) estimated that between 114,000 and 213,000 Puerto Rico residents would leave the island in the year after Hurricane Maria. Using port authority data, Santos (2018) estimated an additional 107,000 Puerto Ricans left in the two months

following the Hurricane. Using flight passenger data Rayer (2018) estimated an increase of 30,000-50,000 Puerto Ricans in Florida.

This case study illustrates the value in collecting large-scale social media data for improving the timeliness of monitoring migration after a natural disaster. Estimates obtained from Facebook data are complementary to official statistics and other methods of dynamic population mapping (Echenique and Melgar, 2018; Meléndez and Hinojosa, 2017; Deville et al., 2014). In particular, cellphone data have been used for mapping population density and changes after crises, with the potential to produce extremely granular estimates of internal migration. However, those data are limited to internal 
movements. Data obtained from Facebook offer a richness of information that allows trends across international borders to be considered, as well as mapping population movements by key demographics such as age and sex. Facebook data and other nontraditional sources are particularly useful for complementing survey data. Social media data will never replace traditional surveys. However, we believe that there are some specific cases, like in the context of natural disasters, where digital breadcrumbs can add relevant information to existing imperfect sources and prove particularly useful, at least in order to generate provisional, but timely, estimates.

There are several limitations of our approach, which are common to all studies that use data from the Facebook Advertising Platform or similar sources (e.g. (Zagheni et al., 2017; Garcia et al., 2018; Rampazzo et al., 2018). Firstly, the users of Facebook are in general not representative of the broader population for which we are interested in obtaining estimates. For example, previous research has shown that Facebook data tends to have a lower proportion of migrants, and a younger age distribution, than the general population (Zagheni et al., 2017). To overcome issues of representativeness, we implemented a difference-in-differences approach, allowing us to assess changes in the Puerto Rican population relative to non-Puerto Rican migrants. Additionally, the magnitude of changes was estimated based on known population sizes in the American Community Survey.

Secondly, we only collected data in three-month intervals, and did not have observations for the months between October and January. We are potentially missing more short-term moves, and underestimating the initial out-migration around the time of the Hurricane. The results presented in this paper offer a further motivation for more frequent data collection in the future, as well as the potential benefit of new and increased collaborations with social media companies such as the 'Social Science One' initiative (Social Science One, 2018).

Lastly, the Facebook data sample displays a level of variability across collection 
waves that appears to be unrelated to migration events. These changes make it more difficult to study substantive changes in migrant populations over time. By using a difference-in-differences approach, we were able to filter out systematic changes in the Facebook data, due for example to changes in the Advertising platform or how the demographics are estimated. However, given the nature of the data and the lack of documentation from Facebook, we may be underestimating our uncertainty. In the future, closer collaborations with social media companies would be important in order to map in a systematic way all potential sources of errors and uncertainty.

Notwithstanding, these results illustrate the power of using social media data to monitor demographic indicators over time, particularly after a shock, such as a natural disaster, to understand large changes in population characteristics. Social media data are especially powerful when coupled with other, complementary, data sources, such as data from traditional surveys and administrative sources (which are representative but often not timely or with sample sizes that are too small for state or local estimates) and mobile phone movements (which are highly granular but do not have demographic information). We found evidence that the majority of Puerto Ricans who left after Hurricane Maria traveled to areas where there was already a large existing population of Puerto Rican migrants, confirming the existence of strong social networks of Puerto Ricans across continental United States. In addition, the observation that the increase in Puerto Rican migrants was disproportionately male and in younger age groups may suggest that the more vulnerable were left behind. This work highlights how rapidly population movements can occur in the months after a natural disaster, and the value in collecting 'digital censuses' to track these short-term changes in populations. 


\section{Notes}

${ }^{1}$ Australia, Austria, Canada, China, France, Germany, Greece, Hungary, India, Indonesia, Ireland, Israel, Italy, Japan, Malaysia, Mexico, Nepal, Philippines, Poland, Portugal, Puerto Rico, Romania, Russia, Singapore, South Korea, Spain, United Arab Emirates, Vietnam. 


\section{References}

Araujo, M., Y. Mejova, I. Weber, and F. Benevenuto (2017). Using Facebook Ads Audiences for Global Lifestyle Disease Surveillance: Promises and Limitations. WebSci '17, New York, NY, USA. ACM.

Deville, P., C. Linard, S. Martin, M. Gilbert, F. R. Stevens, A. E. Gaughan, V. D. Blondel, and A. J. Tatem (2014). Dynamic population mapping using mobile phone data. Proceedings of the National Academy of Sciences 111(45), 15888-15893.

Echenique, M. and L. Melgar (2018, May). Where Puerto Rico's Residents Migrated Since Maria - CityLab.

Fussell, E., N. Sastry, and M. VanLandingham (2010). Race, socioeconomic status, and return migration to New Orleans after Hurricane Katrina. Population and environment 31(1-3), 20-42.

Garcia, D., Y. M. Kassa, A. Cuevas, M. Cebrian, E. Moro, I. Rahwan, and R. Cuevas (2018). Analyzing gender inequality through large-scale Facebook advertising data. Proceedings of the National Academy of Sciences, 201717781.

Kishore, N., D. Marqués, A. Mahmud, M. V. Kiang, I. Rodriguez, A. Fuller, P. Ebner, C. Sorensen, F. Racy, J. Lemery, and others (2018). Mortality in Puerto Rico after Hurricane Maria. New England journal of medicine.

Meléndez, E. and J. Hinojosa (2017). Estimates of post-hurricane María exodus from Puerto Rico. Center for Puerto Rican Studies, Research Brief, October. https://centropr. hunter. cuny. edu/research/data-center/research-briefs/estimatespost-hurricane-maria-exodus-puerto-rico.

Rampazzo, F., E. Zagheni, I. Weber, M. R. Testa, and F. Billari (2018). Mater certa 
est, pater numquam: What can Facebook Advertising Data Tell Us about Male Fertility Rates? arXiv preprint arXiv:1804.04632.

Rayer, S. (2018, October). Estimating the migration of puerto ricans to florida using flight passenger data. URL: https://www.bebr.ufl.edu/sites/default/files/ Research\%20Reports/puerto_rican_migration.pdf.

Rosado, L. (2017, October). Facebook continues to dominate socialnetwork use in Puerto Rico. URL: http://gaitherinternational.com/ facebook-continues-to-dominate-social-network-use-in-puerto-rico/.

Santos, A. (2018). Estimates of excess passenger traffic in Puerto Rico following Hurricane María.

Santos-Burgoa, C., A. Goldman, E. Andrade, N. Barrett, U. Colon-Ramos, M. Edberg, A. Garcia-Meza, L. Goldman, A. Roess, J. Sandberg, and others (2018). Acertainment of the Estimated Excess Mortality from Hurricane Maria in Puerto Rico.

Santos-Lozada, A. R. and J. T. Howard (2018). Use of Death Counts From Vital Statistics to Calculate Excess Deaths in Puerto Rico Following Hurricane Maria. $J A M A$.

Social Science One (2018). Social science one. URL: https://socialscience.one/.

US Census Bureau (2018, October). Us Census Bureau quickfacts: Puerto rico. URL: https://www . census.gov/quickfacts/pr.

Yildiz, D., J. Munson, A. Vitali, R. Tinati, and J. A. Holland (2017). Using Twitter data for demographic research. Demographic Research 37, 1477-1514.

Zagheni, E., V. R. K. Garimella, I. Weber, and others (2014). Inferring international and internal migration patterns from twitter data. In Proceedings of the 23rd International Conference on World Wide Web, pp. 439-444. ACM. 
Zagheni, E., I. Weber, and K. Gummadi (2017). Leveraging Facebook's Advertising Platform to Monitor Stocks of Migrants. Population and Development Review 43(4), $721-734$. 

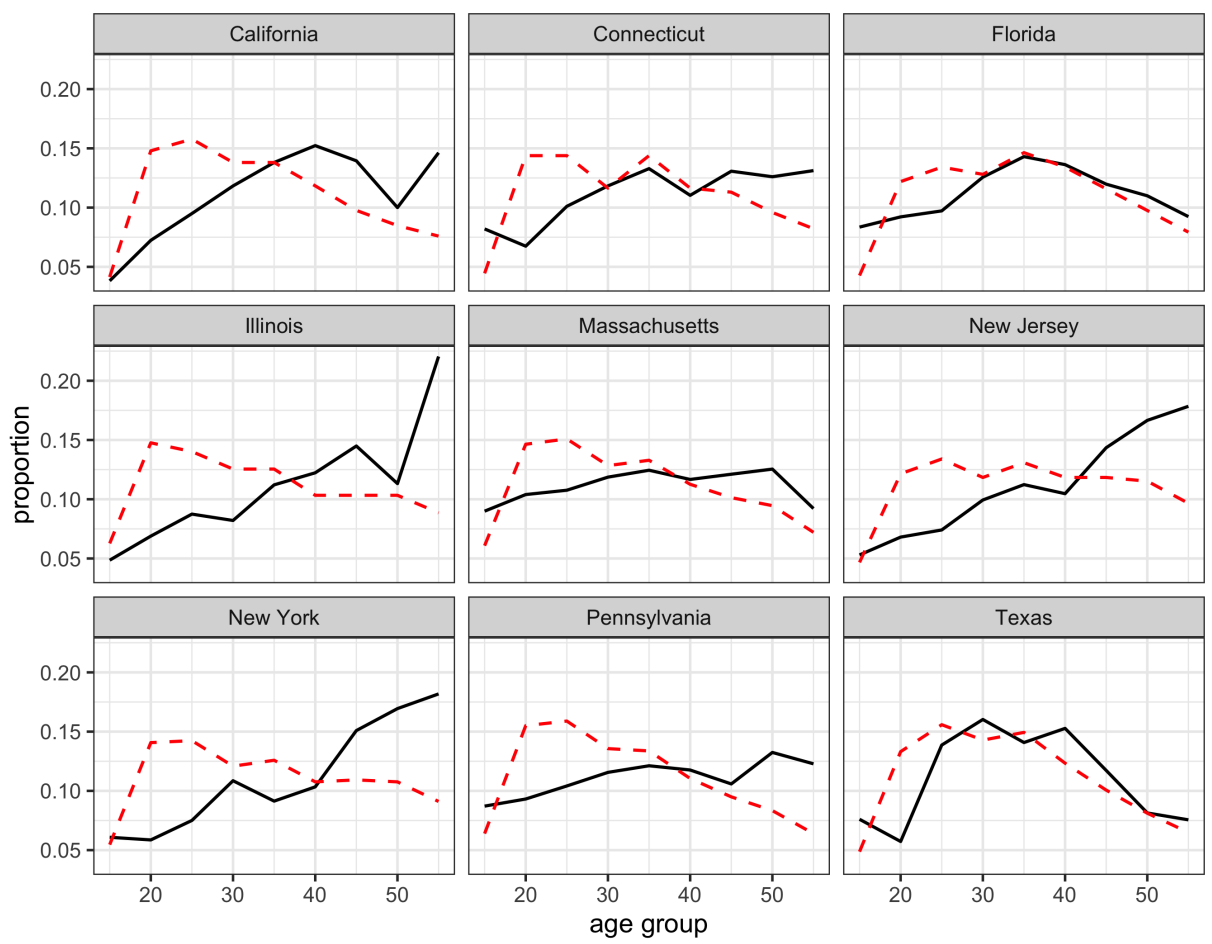

Figure 1: Age distribution of Puerto Rican migrants in Facebook data in January 2017 (red dashed line) and 2016 American Community Survey data (black solid line). 


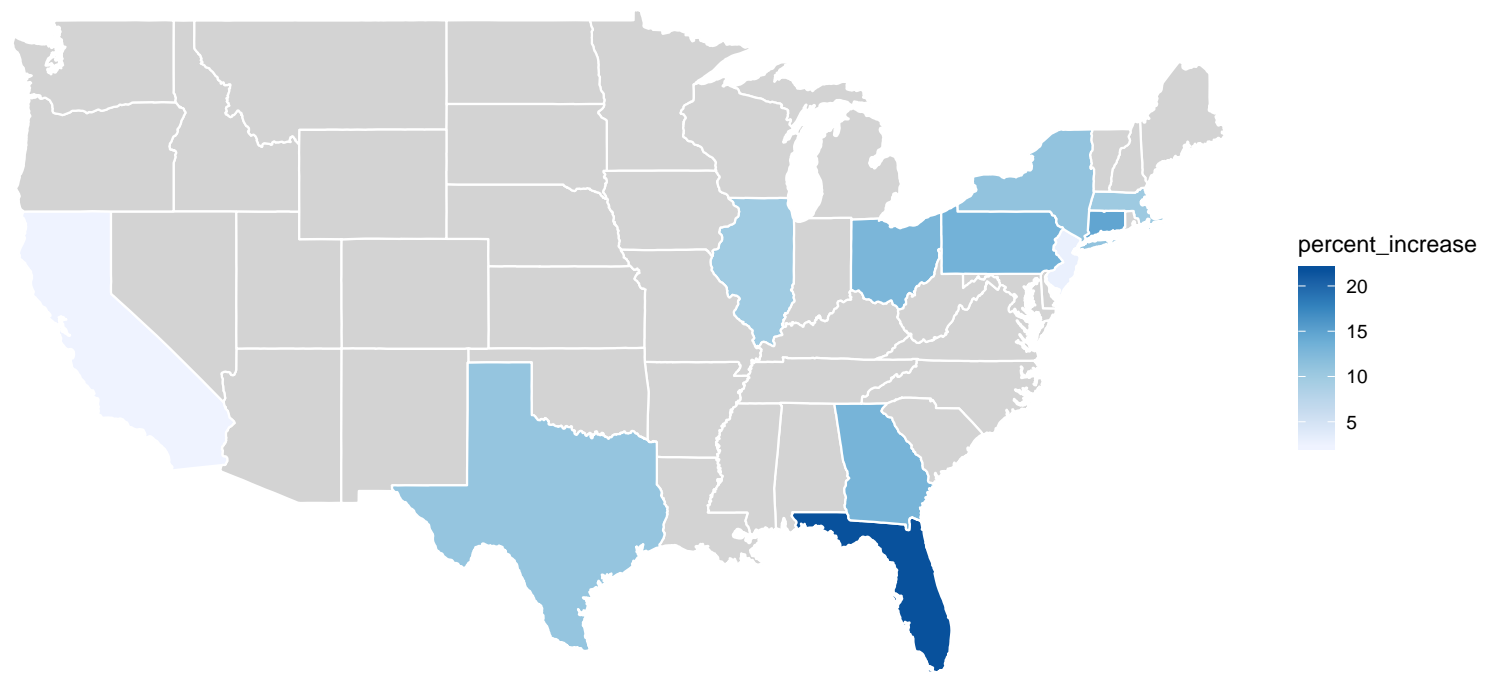

Figure 2: Estimated increase in Puerto Rican migrant stocks from October 2017 to January 2018. Note that only the states with a Puerto Rican migrant population of at least 18,000 are shown. 


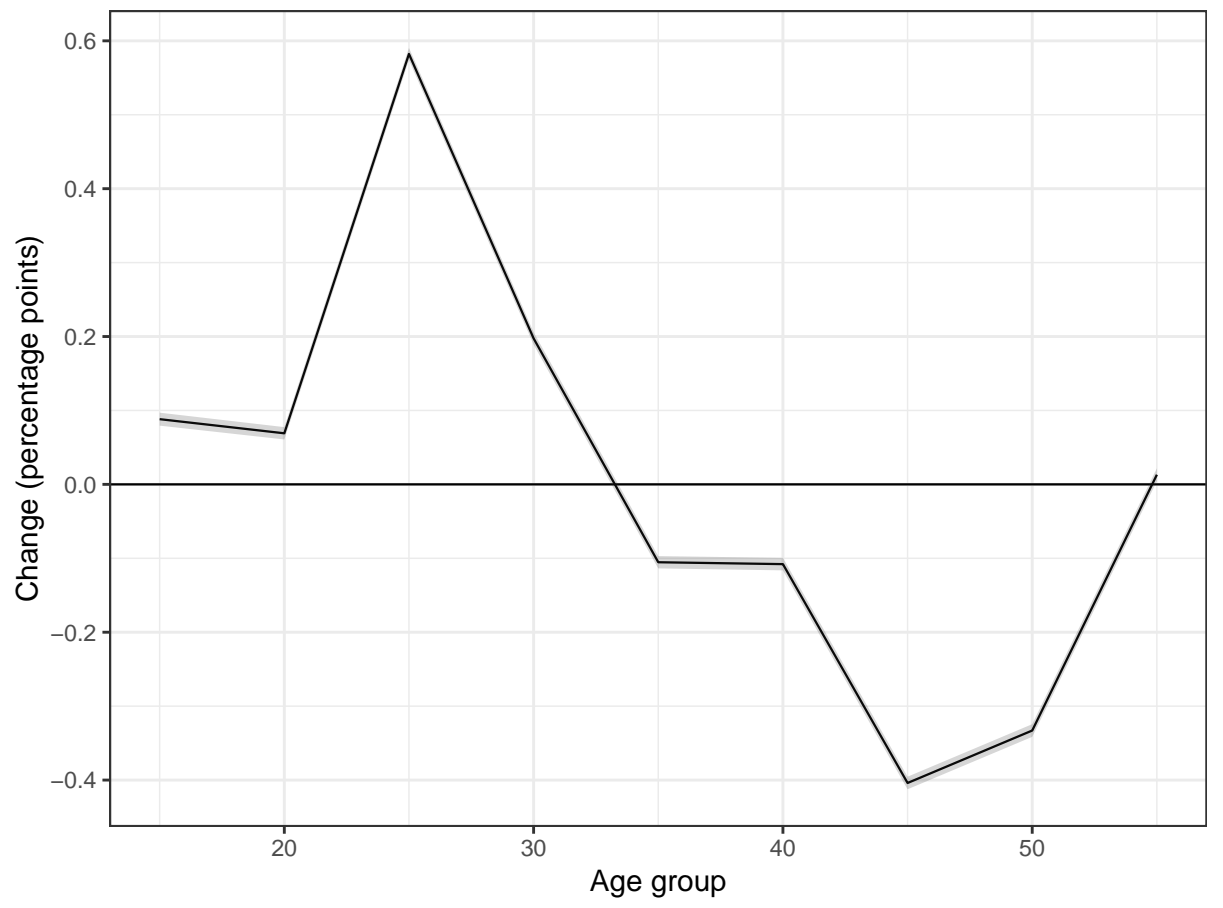

Figure 3: Estimated change in Puerto Rican migrant age distribution from October 2017 to January 2018. 
Table 1: Sex ratio (males to females) in the 2016 American Community Survey and Facebook data in January 2017.

\begin{tabular}{lrr} 
State & ACS sex ratio & Facebook sex ratio \\
\hline California & 1.30 & 0.937 \\
Connecticut & 0.854 & 1.35 \\
Florida & 1.01 & 1.19 \\
Illinois & 1.09 & 1.14 \\
Massachusetts & 0.883 & 1.25 \\
New Jersey & 1.02 & 1.20 \\
New York & 0.905 & 1.19 \\
Pennsylvania & 0.979 & 1.19 \\
Texas & 1.07 & 1.01 \\
\hline
\end{tabular}


Table 2: Estimated increase in Puerto Rican migrant stocks from October 2017 to January 2018. The $95 \%$ confidence intervals are shown in parentheses.

\begin{tabular}{lrr} 
State $(95 \%$ CI $)$ & \% Increase $(95 \%$ CI $)$ & Population Increase \\
\hline Florida & $21.6(20.9,22.3)$ & $65433(63342,67525)$ \\
New York & $11(10.3,11.7)$ & $14477(13584,15371)$ \\
Pennsylvania & $13.4(12.7,14.1)$ & $13441(12700,14181)$ \\
Connecticut & $14.7(12.9,16.5)$ & $9402(8244,10560)$ \\
Massachusetts & $10.1(8.82,11.4)$ & $8957(7824,10090)$ \\
Texas & $10.8(10.4,11.2)$ & $5678(5452,5904)$ \\
Ohio & $12.8(12.2,13.4)$ & $3274(3125,3424)$ \\
Illinois & $9.9(9.15,10.6)$ & $2641(2441,2841)$ \\
Georgia & $13.1(12.4,13.8)$ & $2606(2470,2742)$ \\
New Jersey & $2.9(1.56,4.24)$ & $2282(1228,3336)$ \\
California & $2.4(1.86,2.94)$ & $573(444,702)$ \\
\hline
\end{tabular}


Table 3: Estimated change in Puerto Rican migrant sex ratios (male/female) from October 2017 to January 2018. The 95\% confidence intervals are shown in parentheses.

\begin{tabular}{lrr} 
State & Sex ratio (October 2017$)$ & \% Change (95\% CI) \\
\hline Florida & 1.16 & $0.08(0.076,0.083)$ \\
Texas & 1.01 & $0.065(0.059,0.071)$ \\
Georgia & 1.14 & $0.018(0.006,0.031)$ \\
Connecticut & 1.35 & $0.014(0.004,0.024)$ \\
New Jersey & 1.19 & $0.014(0.005,0.023)$ \\
California & 0.962 & $0.011(0,0.021)$ \\
Massachusetts & 1.28 & $0.008(0,0.016)$ \\
New York & 1.22 & $0.006(0,0.011)$ \\
Ohio & 1.13 & $0.005(-0.007,0.016)$ \\
Illinois & 1.13 & $-0.002(-0.013,0.008)$ \\
Pennsylvania & 1.19 & $-0.053(-0.059,-0.047)$ \\
\hline
\end{tabular}


Table 4: Return migration: Estimated change in Puerto Rican migrant stocks from January 2018 to March 2018. The 95\% confidence intervals are shown in parentheses.

\begin{tabular}{lrr} 
State & \% Change $(95 \% \mathrm{CI})$ & Population Change \\
\hline Florida & $-7.1(-7.77,-6.43)$ & $-21508(-23526,-19490)$ \\
Massachusetts & $-4.5(-5.52,-3.48)$ & $-3991(-4899,-3083)$ \\
Connecticut & $-3.6(-5.04,-2.16)$ & $-2302(-3226,-1379)$ \\
Texas & $-3.5(-3.91,-3.09)$ & $-1840(-2055,-1625)$ \\
Pennsylvania & $-1.4(-2.03,-0.773)$ & $-1404(-2033,-776)$ \\
Ohio & $-1.7(-2.14,-1.26)$ & $-435(-548,-322)$ \\
New York & $0.4(-0.241,1.04)$ & $526(-318,1371)$ \\
Illinois & $2.8(2.15,3.45)$ & $747(574,920)$ \\
Georgia & $6.4(5.86,6.94)$ & $1273(1165,1381)$ \\
New Jersey & $2.3(1.15,3.45)$ & $1810(908,2712)$ \\
California & $8.5(7.97,9.03)$ & $2029(1902,2157)$ \\
\hline
\end{tabular}

